\title{
Paranasal sinus mucoceles, therapeutic solution through external surgical approach
}

\section{Bogdan Mihail Cobzeanu', Dragos Octavian Palade', Gianina Bandol', Patricia Sonia Vonica', Florentina Severin', Liliana Moisii' ${ }^{2}$, Maria Luiza Cobzeanu'², Delia Ciobanu Apostol' ${ }^{3}$, Daniela Viorelia Matei ${ }^{4}$, Mihail Dan Cobzeanu' ${ }^{1}$}

${ }^{1}$ Department of Otorhinolaryngology, "Grigore T. Popa” University of Medicine and Pharmacy, Iasi, Romania

2Department of Radiology and Imagistics, "Grigore T. Popa” University of Medicine and Pharmacy, Iasi, Romania

${ }^{3}$ Department of Morphofunctional Sciences - Pathology, "Grigore T. Popa” University of Medicine and Pharmacy, Iasi, Romania,

${ }^{4}$ Faculty of Medical Bioengineering, "Grigore T. Popa" University of Medicine and Pharmacy, Iasi, Romania

\section{ABSTRACT}

Mucoceles are benign, expansive, cystic tumors, affecting especially the adult, with development in the paranasal sinuses. Clinical symptoms are not specific. These are rare conditions that originate within the sinusal mucosa, favoured by the obstruction of the sinus ostium. Due to the inflammation and the expansive character of the tumor, with the erosion of the bony walls, combined forms can develop, with the involvement of two or more sinuses, most frequently with fronto-ethmoidal localization.

The authors carry out a clinical retrospective study on 25 cases of mucoceles of the paranasal sinuses, diagnosed and treated in the ENT Clinic of the "Sfantul Spiridon" Emergency Clinical Hospital Iasi, during 2015-2019. The authors insist on aspects related to clinical and radiological diagnosis (CT scanner), as well as surgical treatment by external approach.

The postoperative evolution is generally simple, with full recovery, without complications or recurrences.

The diagnosis of the mucoceles of the paranasal sinuses consists in radiologic techniques by preoperative CT scan examination, intraoperative macroscopic aspect and histopathological result.

Surgical approach with complete excision of the tumor and creating a new sinusal drainage path prevents the occurrence of recurrences. KEYWORDS: mucocele, paranasal sinuses mucosa, diagnosis, therapeutic conduct.

\section{INTRODUCTION}

The mucocele is considered a cystic tumor, with a viscous, mucoid content, located at the level of the paranasal sinuses, limited by a membrane, which may expand, eroding the surrounding tissues. It is generally considered to be secondary to an obstruction of the sinus ostium caused by inflammation, fibrosis, tumors or surgery. Bone destruction causes clinical manifestations such as facial swelling, pain, and / or visual disturbances.

Most often, they are located unilaterally at the level of the anterior ethmoid and the frontal sinus, other locations being rare ${ }^{1-6}$.

The content of the mucocele appears as a transparent, mucous, sterile liquid. The infiltration of the bacterial content determines a purulent character, transforming the mucocele into a mucopyocele ${ }^{7}$.

The mucocele may coexist with a sinusitis, confirming the infectious theory, with osteal obstruction and the impossibility of an efficient drainage.

The symptoms of the mucoceles are inconsistent, given their expanding nature, with combined compressive, vasomotor, or dislodging phenom- 
ena, which are conditioned by the topographic location and the evolutionary stage ${ }^{8-10}$.

The radiological examination frequently brings completions and diagnostic clarifications, the CT and MRI images allowing the specification of the mucocele limits ${ }^{11,12}$. The diagnosis confirmation is possible only postoperatively, the treatment of choice being the surgical one by external or endonasal endoscopic approach, trying to marsupialize the mucocele ${ }^{13-16}$.

\section{MATERIAL AND METHODS}

The authors present a retrospective study on a group of 25 cases of mucoceles of the facial sinuses, hospitalized and treated in the ENT Clinic of the "Sfantul Spiridon" Emergency Clinical Hospital, Iasi, between 2015-2019.

The cases included 11 males and 14 females, aged between 21 and 68 years.

The radiological examinations (CT scan) confirmed the localization of the tumors: 15 cases in the frontal sinus, 8 cases with fronto-ethmoidal involvement and one case affecting the maxillary sinus. Four of the cases involving the frontal sinuses presented extension to the orbit, by eroding the superior wall of the orbit, and two cases presented frontal sinusitis.

The symptomatology of these cases was generally nonspecific, and the positive diagnosis was established postoperatively, according to the tumoral macroscopic aspect. Usually, acute or chronic rhinosinusitis does not exclude the coexistence of a mucocele.

The differential diagnosis has to be made with rhinosinusal tumors, pneumosinus dilatans or cysts of the paranasal sinuses.

As a surgical treatment, the mucocele most frequently benefits from an external approach, but lately the possibility of endoscopic resolution by FESS (functional endoscopic sinus surgery) with marsupialization has been discussed. We consider that the external approach is indicated especially in the clinical forms with frontal or fronto-ethmoidal localization, with orbital extension, which require the restoration of the fronto-nasal drainage canal.

The literature discusses endoscopic approach, especially in mucocele variants involving the maxillary sinus. Both the possibility of recurrences after the endoscopic technique, and the certain degree of difficulty in achieving permanent sinus drainage have determined us to support the external surgical approach.

We present the case of a 63-year-old patient who was hospitalized in 2017 for headache, with the appearance of a deformation within the internal

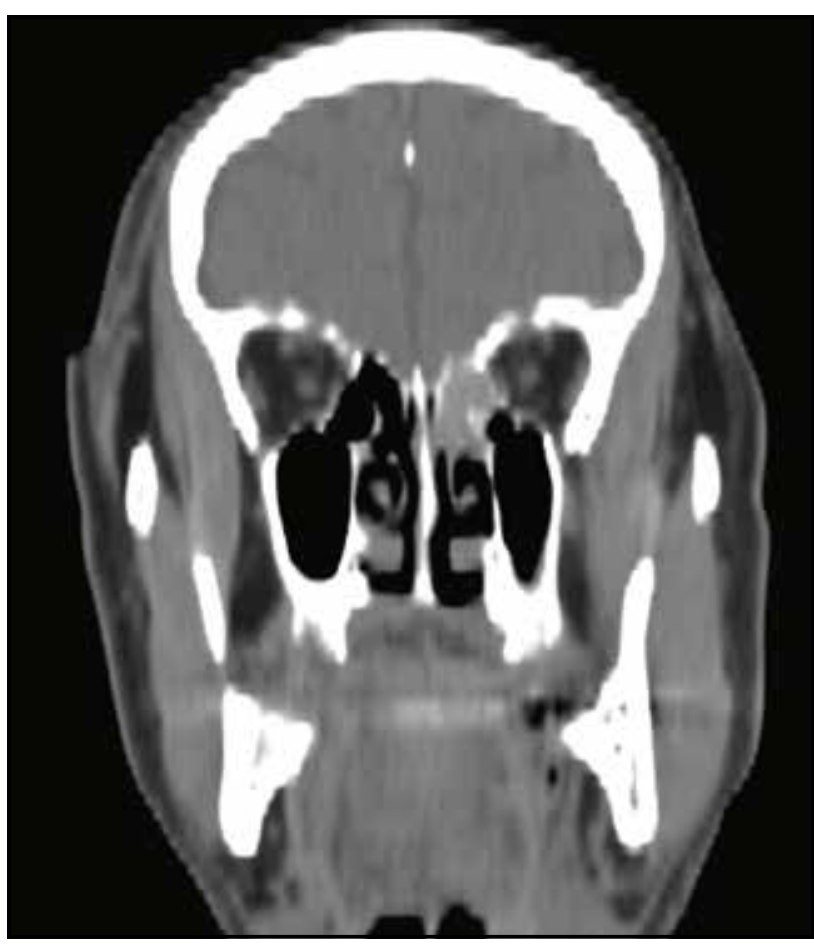

Figure 1. CT of paranasal sinuses (coronal section) - tumor formation in the left frontal sinus and left ethmoid.

angle of the left orbit, pushing the eyeball forward and outward. The patient also complained of the onset of visual problems.

Palpation of the formation revealed an elastic consistency, surrounded by hard, fractured edges determined by the bony edges of the orbit ceiling.

Anterior rhinoscopy revealed a hypertrophy of the middle and lower left turbinates, with a right lateral deviation of the nasal septum and mucosal congestion. Posterior rhinoscopy revealed hypertrophy of the posterior part of the turbinates, with normal aspect of the rhinopharynx.

Ophthalmoscopic examination showed exophthalmia with normal motility of the left eyeball, the fundus and visual acuity being apparently normal.

The CT examination detected a tumor in the left frontal sinus and left ethmoid sinus, with the destruction of the lamina papyracea and the horizontal part of the left frontal sinus, the tumor protruding into the orbit (Figure 1).

In this case, surgery was performed, making an arched incision in the internal angle of the orbit and of the upper left nasogenian groove. Once the tumor and the surrounding bone destruction were exposed, the tumor could be detached from the walls of the frontal and left ethmoid sinuses, suctioning thick, mucous secretions. The cystic tumor was excised and sent for histopathological examination.

Bone erosion of the ethmoid and the lower wall 


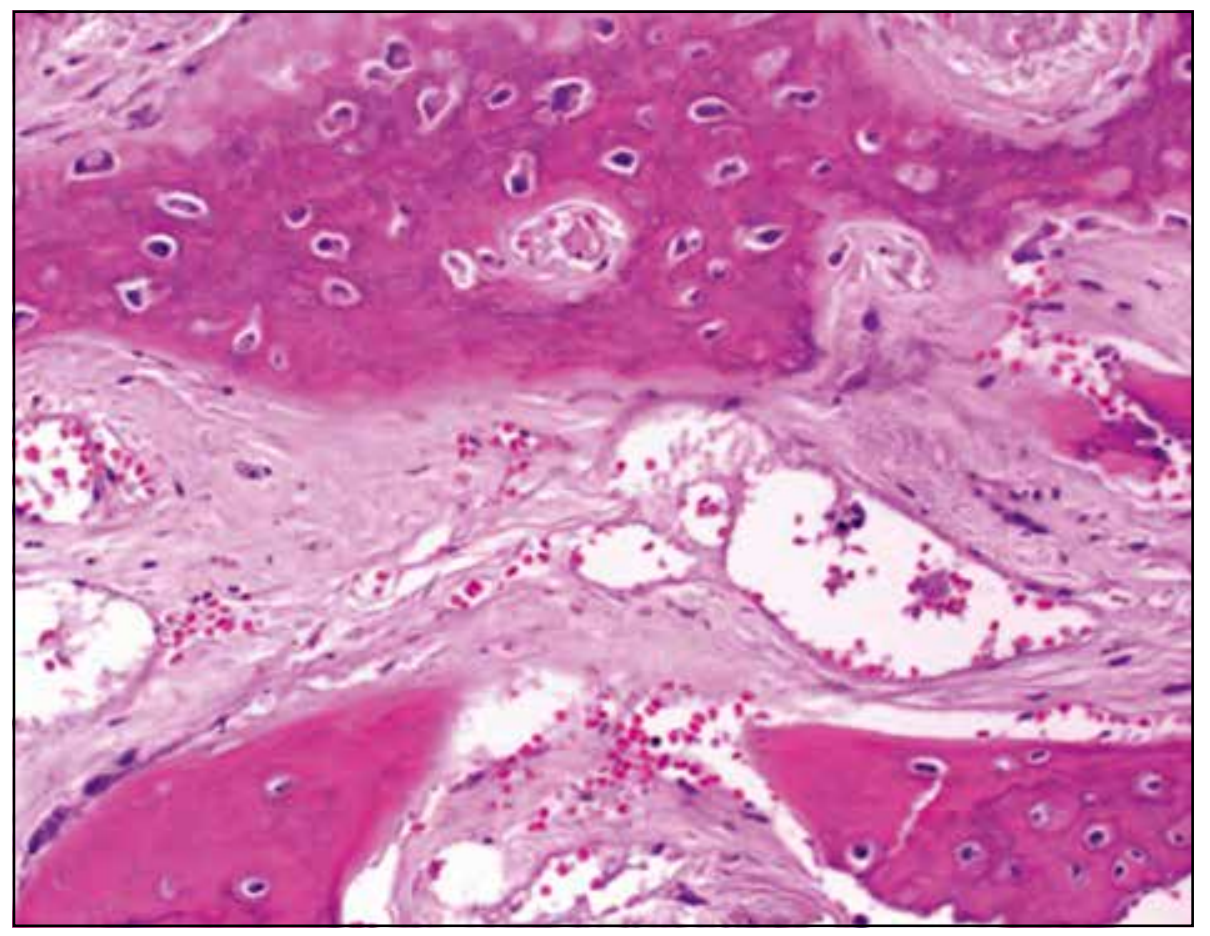

Figure 2. Cyst wall lined by a simple flattened epithelium with abundant pale eosinophilic mucus, that associates numerous macrophages with foamy cytoplasm; $\mathrm{HE}, \mathrm{x} 4$.

of the left frontal sinus is characteristic for this type of tumor and the histopathological exam confirmed the clinical and intraoperative diagnosis of left fronto-ethmoidal mucocele (Figure 2).

A left fronto-nasal neo-canal was made, and a silicone drain was left in place for 3 weeks to prevent stenosis (Figures 3-8).

The postoperative evolution was favourable, without local recurrence or other complications.

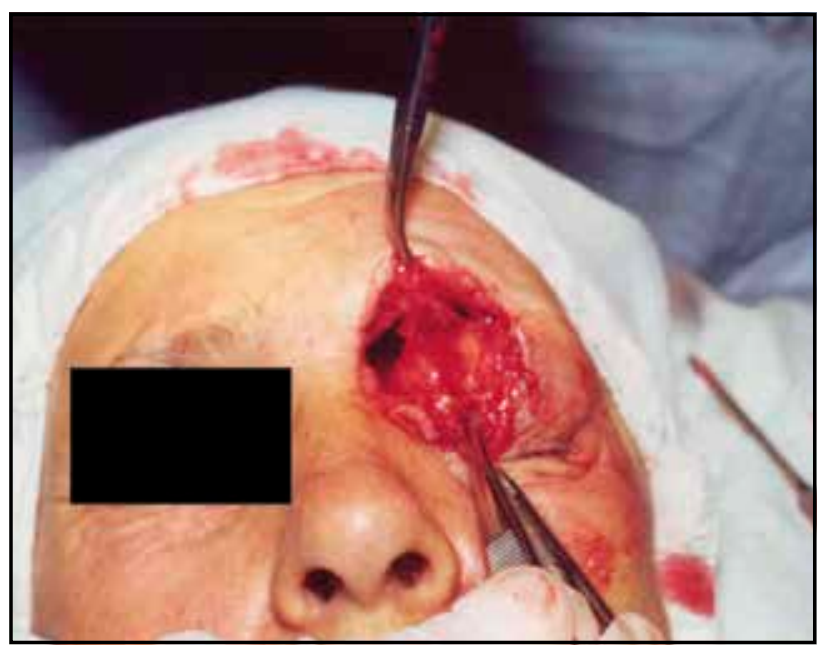

Figure 3. Arciform incision in the inner angle of the orbit and of the upper left nasogenian groove.

\section{RESULTS AND DISCUSSIONS}

We consider that surgery is the treatment of choice in solving the mucoceles of the paranasal sinuses, with the ablation of the cystic tumor and creating an efficient drainage path; otherwise, the recurrence is almost constant. No recurrences were observed in our case, and by maintaining the drain tube for 3 weeks, the stenosis of the sinus drainage path was avoided.

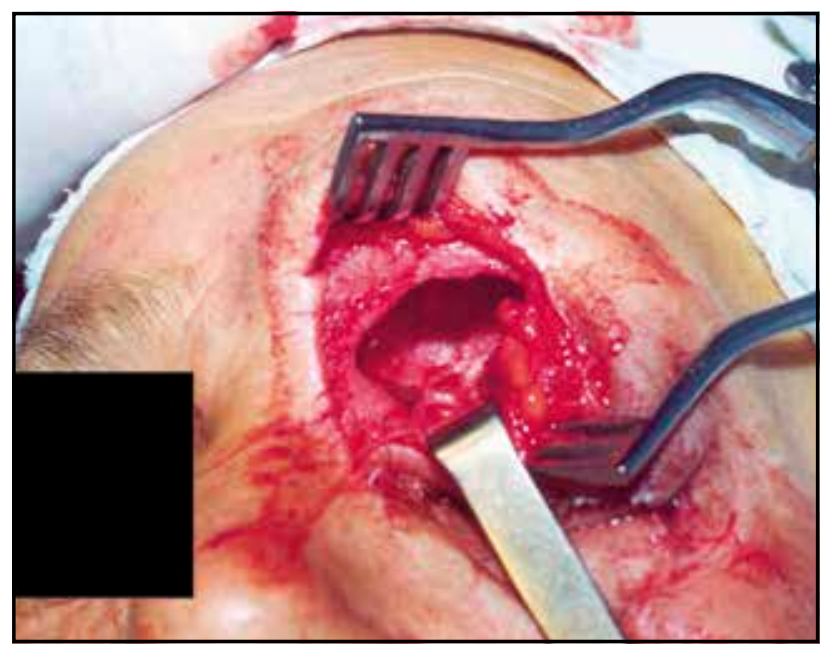

Figure 4. The appearance of the mucocele with surrounding bone destruction. 




Figure 5. Local appearance after the mucocele excision.

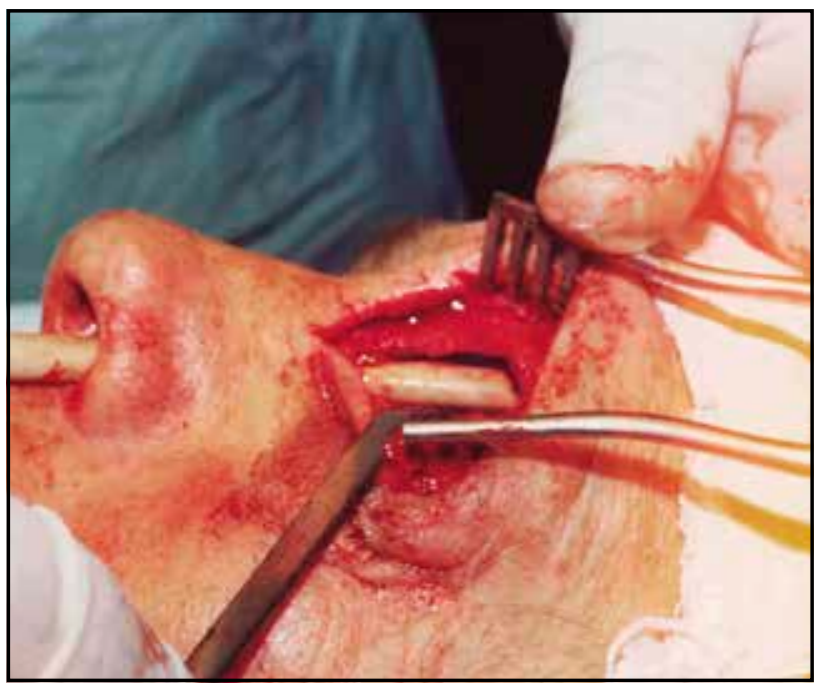

Figure 7. Drainage of the new fronto-nasal canal.

In case of mucopyoceles, drainage and antibiotic therapy according to the antibiogram allowed their resolution. In one case of fronto-ethmoidal mucocele, the preservation of a portion of the mucocele wall required the practice of marsupialization. It was made by a wide opening on the outside and a suture of the mucocele pouch.

\section{CONCLUSIONS}

A mucocele is a benign tumor developed from the mucosa of the paranasal sinuses, most often favoured by sino-nasal inflammation and obliteration of the sinus drainage pathway. Its symptomatology is often determined by the expansion and compression of the surrounding ele-

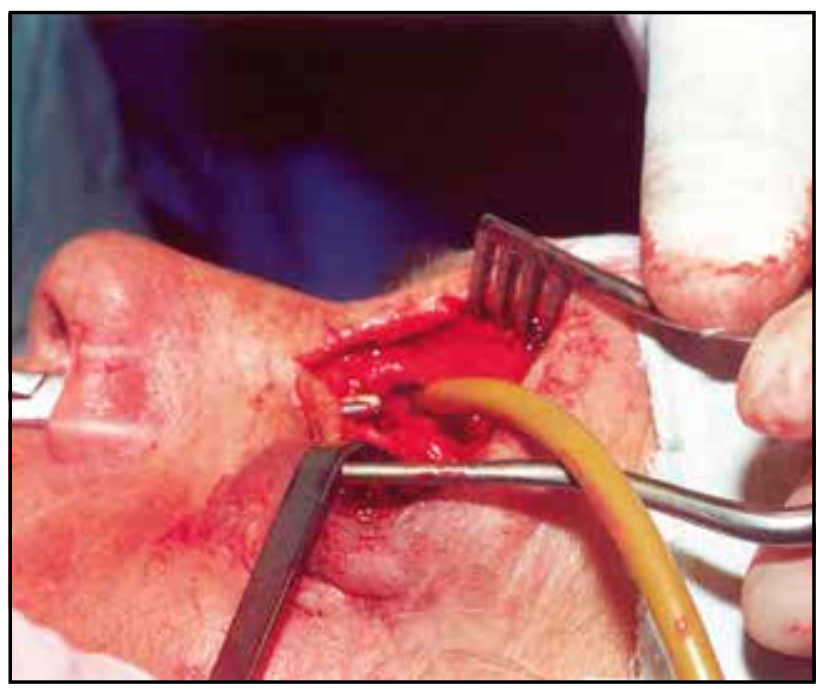

Figure 6. Creating the new fronto-nasal canal.

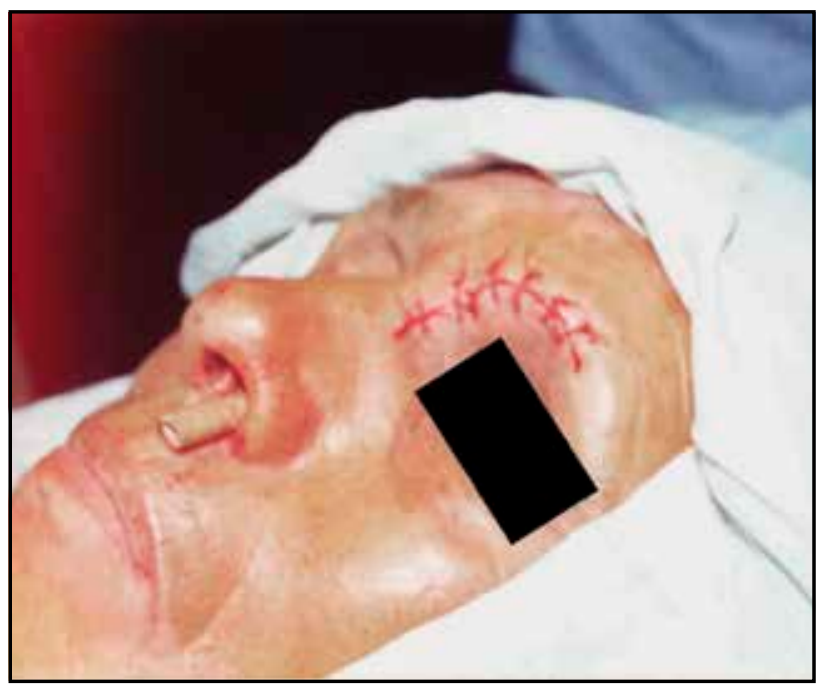

Figure 8. Creating the new fronto-nasal canal.

ments, having ophthalmological, neurological and rhinological implications.

The use of CT or MRI examination is useful in determining the extension of the mucocele at the orbital and endocranial level, showing the possibility of compartmentation, as well as bone destruction.

The therapeutic solution is exclusively surgical, by external or endoscopic approach with the removal or marsupialization of the tumor and the achievement of an efficient, permanent drainage to the nasal fossa.

Conflict of interest: The authors declare there is no conflict of interest.

Contribution of authors: All the authors have equally contributed to this work. 


\section{REFERENCES}

1. Lund VJ, Milroy CM. Fronto-ethmoidal mucocoeles: a histopathological analysis. J Laryngol Otol. 1991;105(11):921-3. DOI: 10.1017/ s0022215100117827.

2. Qureishi A, Lennox P, Bottrill I. Bilateral maxillary mucoceles: an unusual presentation of cystic fibrosis. J Laryngol Otol. 2012;126(3):319-21. DOI: $10.1017 / \mathrm{S} 002221511100329 \mathrm{X}$.

3. Yilmaz F, Karaaslan K, Yigit B, Gurel K, Yilmaz F. Giant mucocele of the maxillary sinus: a case report. KBB-Forum. 2008;7:111-3.

4. Thompson LDR. Paranasal sinus mucocele. Ear Nose Throat J. 2012;91:276-8.

5. Devars du Mayne M, Moya-Plana A, Malinvaud D, Laccourreye O, Bonfils P. Sinus mucocele: natural history and long-term recurrence rate. Eur Ann Otorhinolaryngol Head Neck Dis. 2012;129(3):125-30. DOI: 10.1016/j.anorl.2011.10.002.

6. Uysal IO, Yuce S, Kosger H, Müderris S. Maxillary sinus mucocele: report of a case. KBB BBC Derg. 2003;11:77-80.

7. Aycicek A, Sargin R, Yilmaz D, Temiz M, Kenar F, Yildiz H. Frontal sinus mucocele: a case report. Med J Kocatepe. 2008:17-20.

8. Har-El G, Balwally AN, Lucente FE. Sinus mucoceles: is marsupialization enough? Otolaryngol Head Neck Surg. 1997;117(6):633-40. DOI: 10.1016/s0194-5998(97)70045-7.

9. Capra GG, Carbone PN, Mullin DP. Paranasal sinus mucocele. Head Neck Pathol. 2012;6(3):369-72. DOI: 10.1007/s12105-012-0359-2.

10. Arrue P, Kany MT, Serrano E, Lacroix F, Percodani J, Yardeni E, et al. Mucoceles of the paranasal sinuses: uncommon location. J Laryngol Otol. 1998;112(9):840-4. DOI: 10.1017/s0022215100141854.

11. Lloyd G, Lund VJ, Sany L, Howard D. Optimum imaging for mucoceles J Laryngol Otol. 2000;114(3):233-6. DOI: 10.1258/0022215001905238.

12. Carvalho BV, Lopes ICC, Correa JB, Ramos LFM, Motta EGPC, Diniz RLFC. Typical and atypical presentations of paranasal sinus mucocele at computed tomography. Radiol Bras. 2013;46:372-5.

13. Lawson W. Frontal sinus. In: Blitzer A, Lawson W, Friedmann WH. Surgery of the Paranasal Sinuses. Philadelphia P.A.: WB Saunders Co; 1991, p.183-218.

14. Ianneti G, Cascone P, Valentini V, Agrillo A. Paranasal sinus mucocele: diagnosis and treatment. J Craniofac Surg. 1997;8(5):391-8.

15. Beasley NJP, Jones NS. Paranasal sinus mucoceles: modern management. Am J Rhinology. 1995;9(5):251-6.

16. Bockmühl U, Kratzsch B, Benda K, Draf W. Paranasal sinus mucoceles: surgical management and long term results. Laryngorhinootologie. 2005;84(12):892-8. DOI: 10.1055/s-2005-870572. 